\title{
Tratamento dos aneurismas da aorta descendente por endoprótese (tromba de elefante)
}

\author{
Honório PALMA*, João Aléssio JULIANO*, Ruy Guilherme Rodrigues CAL*, Dirceu Rodrigues de ALMEIDA*, \\ Luiz Horitoshi OTA*, Inês Abrantes GIANOTTI*, José Carlos S. ANDRADE*, Ênio BUFFOLO*
}

RBCCV 44205-92

\begin{abstract}
PALMA, H.; JULIANO, J. A.; CAL, R. G. R.; ALMEIDA, D. R.; OTA, L. H.; GIANOTTI, I. A.; ANDRADE, J. C. S.; BUFFOLO, E. - Tratamento dos aneurismas da aorta descendente por endoprótese (tromba de elefante). Rev. Bras. Cir. Cardiovasc., 4(3): 190-194, 1989.

RESUMO: Os aneurismas da aorta descendente ainda causam grande mortalidade e morbidade, apesar dos avanços da cirurgia cardiovascular moderna. Neste trabalho utilizamos, originalmente em nosso meio, uma técnica recente que consiste na inserção de uma prótese de Dacron intraluminar, suturada apenas em sua porção proximal, logo após a subclávia esquerda e que transpōe a zona do aneurisma, ficando solta no interior da aorta em sua porção distal (tromba de elefante). A operação é realizada com circulação extracorpórea, hipotermia profunda de $19^{\circ} \mathrm{C}$, com parada circulatória no tempo suficiente para a inserção da prótese e sutura da sua borda proximal. Desde maio de 1988, oito pacientes fizeram parte deste estudo, sendo que seis eram dissecçōes da aorta tipo B e dois casos, aneurismas verdadeiros. A simplicidade e rapidez do procedimento, assim como os resultados pós-operatórios obtidos nos animam a prosseguir e recomendar este tipo de alternativa tática, especialmente nas dissecçōes de aorta tipo B.
\end{abstract}

DESCRITORES: aneurismas de aorta descendente, cirurgia; endoprótese.

\section{INTRODUÇÃO}

Diferentes métodos foram e são utilizados para o tratamento das lesões da aorta descendente desde o simples acompanhamento clínico, até as técnicas operatórias mais complexas que visam substituir a aorta doente por tubos artificiais. Para a obtenção deste objetivo, utiliza-se desde pinçamento simples da aorta com hipotensão controlada ', até desvios circulatórios temporários, tais como: shunts extra-anatômicos, desvios átrio esquerdo-femoral ou veno-arterial, utilizando-se, nesta última situação, um oxigenador artificial.

Estas técnicas exigem dissecçāo da lesāo aórtica, separando-a das estruturas vizinhas na obtenção dos cotos proximal e distal e que constitui procedimento trabalhoso, demorado e por vezes arriscado.
BORST et alii ${ }^{2,3}$ originalmente descrevem uma tática operatória na correção de aneurismas da aorta que envolvia mais de uma porção da mesma, na qual o aneurisma da croça era tratado primariamente com a interposição de um enxerto de Dacron e a extremidade distal do tubo deixada solta na aorta descendente, para uma correção total num segundo tempo operatório.

Verificou-se, nos casos reestudados, que o tubo aderia firmemente à parede aórtica, dispensando a necessidade da reoperação, em alguns casos.

Entusiasmados com a simplicidade deste procedimento e baseados na alta mortalidade verificada no tratamento cirúrgico das dissecçōes da aorta tipo $B{ }^{4}$, resolvemos realizar, originalmente, o implante da endoprótese

\footnotetext{
Trabalho realizado no Hospital São Paulo. Escola Paulista de Mecicina. São Paulo, SP, Brasil. Apresentado ao 16: Congresso Nacional de Cirurgia Cardiaca. Sảo Paulo, 7 e 8 de abril, 1989.

- Do Hospital Sâo Paulo. Escola Paulista de Medicina.

Endereço para separatas: Rua Borges Lagoa, 783. 5 andar. 04038 São Paulo, SP, Brasil
} 
PALMA, H.; JULIANO, J. A.; CAL, R. G. R.; ALMEIDA, D. R.; OTA, L. H.; GIANOTTI, I. A.; ANDRADE, J. C. S.; BUFFOLO, E. - Tratamento dos aneurismas da aorta descendente por endoprótese (tromba de elefante). Rev. Bras. Cir. Cardiovasc., 4(3): 190-194, 1989

sob hipotermia profunda e parada circulatória para as dissecçōes deste tipo.

\section{CASUISTICA E MÉTODOS}

Foram operados oito pacientes, de maio de 1988 a fevereiro de 1989 , sendo que todos eram do sexo masculino e com idades variando de 46 a 79 anos. O início dos sintomas era recente e, em todos os casos que evoluiam com aneurismas verdadeiros, todos apresentavam complicações pré-operatórias, como podemos observar na Tabela 1. Dos oito pacientes estudados, seis eram portadores de dissecção aórtica aguda do tipo $\mathrm{B}$ e dois, portadores de aneurisma verdadeiro em expansāo aguda.

Em relação ao diagnóstico, o cateterismo cardiaco com aortografia, o eco-Doppler e a tomografia computadorizada constratada foram realizados em todos os casos.

O princípio da técnica está ilustrado esquematicamente na Figura 1. Por esternotomia longitudinal, a circulaçāo extracorpórea foi instalada com duas cânulas, uma de $1 / 2$ polegada no átrio direito e outra na aorta ascendente. A parede circulatória foi estabelecida logo depois de atingirmos a temperatura de $19-20^{\circ} \mathrm{C}$ pelo termômetro nasofaríngeo e procedendo, então, à incisão longitudinal na croça da aorta em sua parede anterior. Inspecionada a aorta, procuramos identificar a lesão distalmente à subclávia esquerda.

A extensāo da prótese foi determinada analizando-se os exames pré-operatórios, especialmente a tomo-

TABELA 1

Idade Antecedentes Complicaçōes pré-operatónias

\begin{tabular}{llll}
\hline 1 & 64 & Hipertensão & Hemoptise \\
\hline 2 & 79 & $\begin{array}{l}\text { Hipertensão } \\
\text { Alcoolismo }\end{array}$ & $\begin{array}{l}\text { Derrame Pleural Esquerdo } \\
\text { Hemorragia Digestiva } \\
\text { Hipotensão Arterial }\end{array}$ \\
\hline 3 & 64 & $\begin{array}{l}\text { Hipertensão } \\
\text { Tabagismo }\end{array}$ & $\begin{array}{l}\text { Paresia de Membro Inferior Esquerdo } \\
\text { Retenção Urinária } \\
\text { Tumor Abdominal }\end{array}$ \\
\hline 4 & 50 & Hipertensão & $\begin{array}{l}\text { Isquemia Aguda do Membro Inferior } \\
\text { Esquerdo }\end{array}$ \\
\hline 5 & 43 & $\begin{array}{l}\text { Hipertensão } \\
\text { Tabagismo }\end{array}$ & $\begin{array}{l}\text { Hematúria } \\
\text { Edema de Membro Superior Esquerdo } \\
\text { Por Trombose da Sublcávia Esquerda }\end{array}$ \\
\hline 6 & 56 & $\begin{array}{lll}\text { Hipertensão } \\
\text { Derrame Pleural Bilateral } \\
\text { Pneumonia }\end{array}$ \\
\hline 7 & 62 & $\begin{array}{l}\text { Hipertensão } \\
\text { Diabetes }\end{array}$ & Hemoptise \\
\hline 8 & 54 & Hipertensão & Hemoptise \\
\hline
\end{tabular}

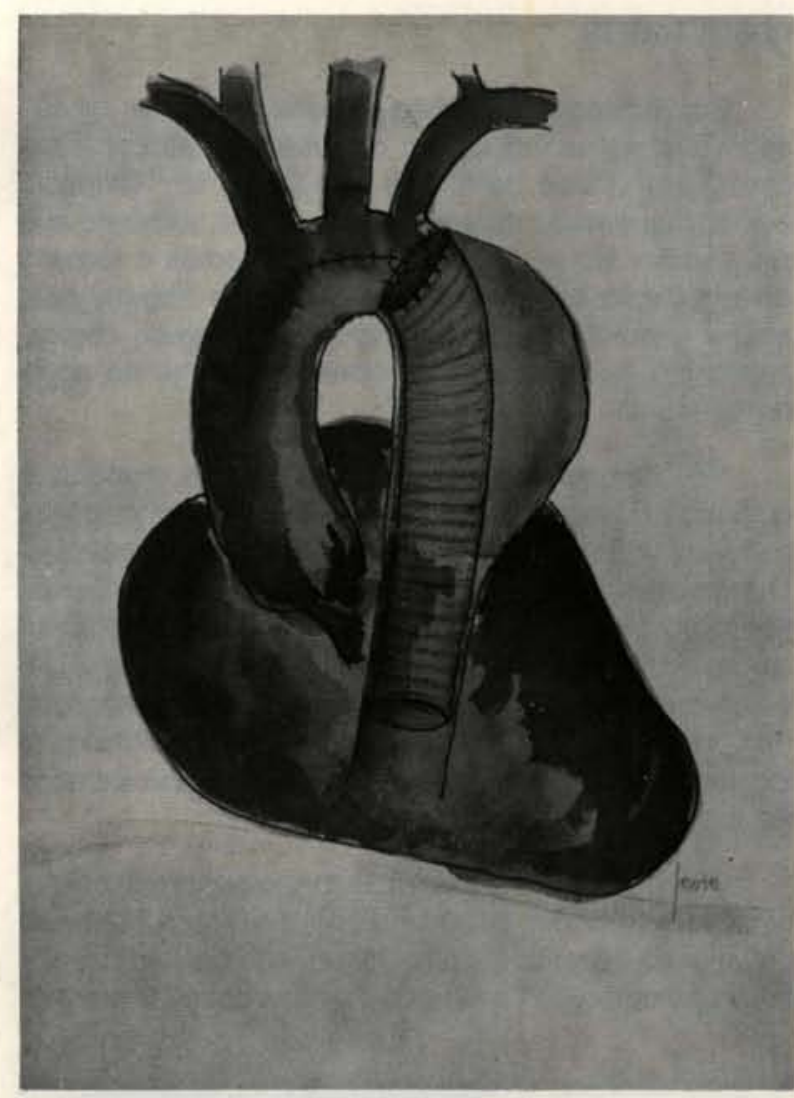

Fig. 1 - Representação esquemática da endoprótese.

grafia, sendo que o seu diâmetro foi escolhido a partir da circunferência interna da aorta ao nível da subclávia esquerda, com obturadores aferidos. A seguir, a prótese foi introduzida distalmente com manobras digitais e suturada com prolene 3-0 somente em borda proximal logo após a emergência da artéria subclávia esquerda. No último caso, foi utilizado um recurso adicional de introdução de um broncofibroscópio na luz da aorta descendente, com o objetivo de aferir mais corretamente o comprimento e a posição da prótese ao final do implante.

A circulação extracorpórea foi restaurada lentamente, procurando dar saída ao ar pela incisão da croça da aorta e também pela artéria femoral, neste tempo aberta.

Durante a parada circulatória, o tronco braquiocefálico e a carótida esquerda permaneceram ocluídos pela aplicação de garrotes.

Após o aquecimento à temperatura fisiológica, e estando os parâmetros hemodinâmicos estáveis, a circulação extracorpórea foi descontinuada, sendo retirados os tubos e neutralizada a heparinemia. 
PALMA, H.; JULIANO, J. A.; CAL, R. G. R.; ALMEIDA, D. R.; OTA, L. H.; GIANOTTI, I. A.; ANDRADE, J. C. S.; BUFFOLO, E. - Tratamento dos aneurismas da aorta descendente por endoprótese (tromba de elefante). Rev. Bras. Cir. CardiovasC., 4(3): 190-194, 1989.

\section{RESULTADOS}

Em relação à operação, encontramos uma dificuldade relativa na introdução da prótese, nos primeiros casos, em virtude de o tubo de Dacron ter tendência a aderir na parede da aorta descendente, evitando sua progressão. No primeiro paciente, utilizamos o recurso da introdução de um calibroso cateter de Fogarty pela artéria femoral, que foi exteriorizado na croça aórtica, auxiliando na colocação apropriada do tubo na aorta descendente.

Nos demais pacientes, com a seleção apropriada do calibre do tubo e com o auxilio de manobras digitais, conseguimos sua introdução sem maiores dificuldades. O tempo cirúrgico total foi, em média, de cinco horas, sendo o tempo médio de parada circulatória de 39 minutos, com tendência a diminuição nos últimos casos. Um dos pacientes, devido ao achado cirúrgico de dissecção retrógrada, o que não correspondia ao diagnóstico prévio, foi a óbito no centro cirúrgico, por perfusão da falsa luz e isquemia miocárdica.

O sangramento observado no pós-operatório imediato, foi, em média, de $390 \mathrm{ml}$ e três pacientes morreram na unidade de terapia intensiva, por causas não relacionadas ao emprego do método e sim às condiçōes pré-o-

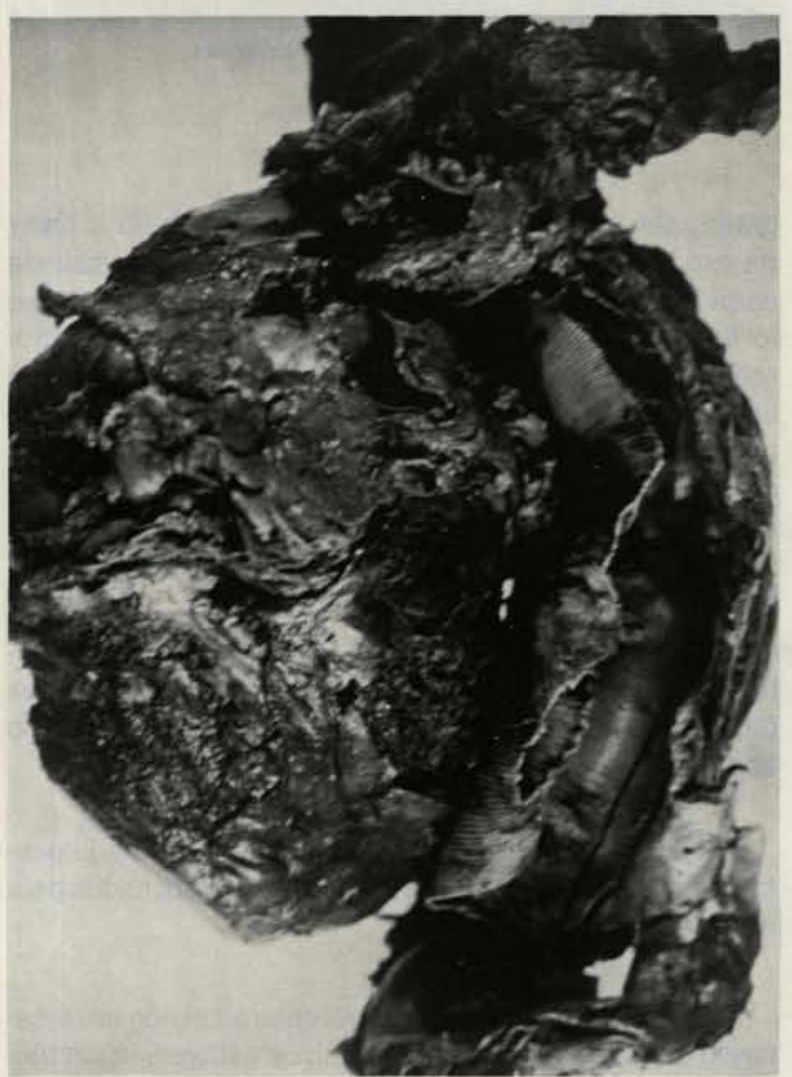

Fig. 2 - Peça da necropsia. Tubo de Dacron no interior da aorta descendente.

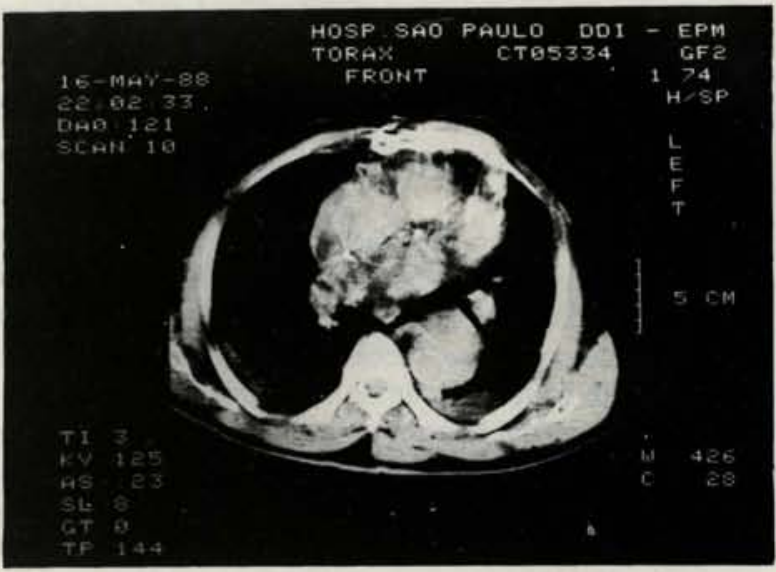

Fig. 3 - Tomografia computadorizada no pós-operatório.

peratórias. A hipóxia grave por broncoespasmo foi a causa do óbito no terceiro dia em um caso; a insuficiência de múltiplos órgãos ocorrida no 26 : dia em outro e, por fim, a isquemia abdominal associada a isquemia de membro inferior esquerdo em outro.

Realizamos necropsia em dois destes casos e observamos que as próteses estavam aderidas à parede da aorta e não se encontravam dobradas (Figura 2).

Os quatro pacientes em seguimento, em nosso Serviço, são submetidos, periodicamente, a tomografia computadorizada, a qual tem demonstrado uma boa evolução com as próteses bem localizadas e com trombose organizada em regressão ao redor da prótese, não se observando falsa luz (Figura 3 ).

Sintomas observados no pré-operatório, tais como escarro hemoptóico (pacientes 1 e 7), desapareceram e o edema do membro superior esquerdo por trombose da subclávia (paciente 5) regrediu acentuadamente.

\section{DISCUSSÃO}

A utilização deste método restringe-se, a nosso ver: 1) às dissecçōes limitadas à aorta descendente, desde que a soluçăo de continuidade da íntima se localize logo no início desta porçāo; 2) aos aneurismas verdadeiros que se iniciam junto à subclávia esquerda e que não se extendem por toda a aorta descendente, sendo o coto proximal de difícil abordagem pela toracotomia esquerda convencional. Lembramos, também, que, nos casos em que houver rotura como complicação, por não suturarmos a porção distal da prótese, esta técnica, pela lógica, não deve ser utilizada.

O acesso cirúrgico feito por esternotomia, que é - mais utilizado na rotina da cirurgia cardíaca, permite um controle total sobre as condições do paciente e, além disso, por essa via não temos contato direto com o aneurisma, evitando as dificuldades técnicas inerentes à cirur- 
PALMA, H.; JULIANO, J. A.; CAL, R. G. R.; ALMEIDA, D. R.; OTA, L. H.; GIANOTTI, I. A.; ANDRADE, J. C. S.; BUFFOLO, E. - Tratamento dos aneurismas da aorta descendente por endoprótese (tromba de elefante). Rev. Bras. Cir. CardiovasC., 4(3): 190-194, 1989

gia tradicional, como, por exemplo, a dissecção das estruturas aderidas, a necessidade de pinçamento proximal e distal ao aneurisma e o sangramento das artérias intercostais.

Durante o implante da prótese, sempre nos preocupou o tamanho da mesma o e seu posicionamento no interior da aorta e, apesar dos bons resultados demonstrados pelos exames pós-operatórios e pelo material de necropsia, realizamos um treinamento em cadáveres em que a face interna da aorta descendente foi examinada com o broncofibroscópio. O objetivo desse exame foi determinar, não só a distância entre a saída da subclávia esquerda e o final da parede doente, como também a não existência de dobras na prótese após sua colocação. Devemos ressaltar, também, que o tempo cirúrgico foi menor do que aquele observado com as técnicas tradicionais e, ainda, que a perda sangüínea, tanto no intra-operatório como no pós-operatório, foi pequena graças à pouca necessidade de dissecção.

RBCCV 44205-92

PALMA, H.; JULIANO, J. A.; CAL, R. G. R.; ALMEIDA, D. R.; OTA, L. H.; GIANOTTI, I. A.; ANDRADE, J. C. S.; BUFFOLO, E. - Treatment of the aneurysms of descending aorta for intraluminal prosthesis (elephant trunk). Rev. Bras. Cir. Cardiovasc., 4(3): 190-194, 1989.

ABSTRACT: Aneurysms of descending aorta still cause great morbidity and mortality even in modern cardiac surgery days. In this paper, we describe the use of a recente technique which consists in the insertion of an intraluminal Dacron prosthesis, sutured only in its proximal end, just after the left subclavian artery. The prosthesis goes beyoud the aneurysm area and stays free in its distal aortic segment (elephant trunk). The operation is performed with bypass and profound hypothermia at $19^{\circ} \mathrm{C}$ with circulatory arrest for just enough time to insert the prosthesis and make the proximal suture. Since May 1988 eight patients underwent this kind of surgery: six cases of acute type B dissection and two cases of saccular aneurysms. The procedure is very simple and fast and the post-operative results obtained make clear that our experience should go on. We recommend this procedure as a tactical alternative, specially for type B dissections.

DESCRIPTORS: aneurysms of descending aorta, surgery; intraluminal prosthesis.

\section{REFERÊNCIAS BIBLIOGRÁFICAS}

1 CRAWFORD, E. S. \& CRAWFORD, J. S. - Diseases of the aorta: including an atlas of angiographic pathology and surgical technique. Baltimore, Williams \& Wilkins, 1984. p. 37-39.

2 BORST, H. G.; FRANK, G.; SCHAPS, D. - Treatment of extensive aortic aneurysms by a new multiple stage approach. J. Thorac. Cardiovasc. Surg., 95: 11-13, 1988.

3 BORST, H. G.; WALTERBUSCH, G.; SCHAPS, D. - Extensive aortic replacement using "elephant trunk" prosthesis. Thorac. Cardiovasc. Surg., 31: 37-40, 1983.

4 DAILY, P. O.; TRUEBLOOD, H. W.; STINSON, E. B.; WUERFLELIN, R. D.; SHUMWAY, N. E. - Management of acute aortic dissections. Ann. Thorac. Surg., 10: 237,1970

\section{Discussão}

\section{DR. OSWALDO TENO CASTILHO Ribeiräo Preto, SP}

O trabalho apresentado pelos autores mostra, novamente, as dificuldades existentes no tratamento cirúrgico das dissecçōes agudas de aorta, evidenciando a grande diferença que existe entre a abordagem cirúrgica na fase aguda, onde a mortalidade é altíssima, e a abordagem na fase crônica, de melhor prognóstico. Em razão disto, traz novamente as variações técnicas, com um novo tipo de cirurgia paliativa, a exemplo da cirurgia de Carpentier, cirurgia de fenestração e outras. Concordamos em grande parte com as consideraçōes apresentadas pelo Dr. Palma e o grupo da Escola Paulista de Medicina, baseado numa casuística de nosso Serviço na Santa Casa de Ribeirão Preto, de 72 casos de D.A., estudados e tratados realmente na fase aguda. Preocupados também com as dificuldades técnicas da abordagem direta da lesão, publicamos uma técnica pessoal, baseada na técnica da fenestração de De Bakey, para tratamento cirúrgico das dissecções agudas tipo B (ou tipo III de De Bakey), que descrevemos a seguir. Operamos 16 pacientes com esta técnica, com seis óbitos. O grande problema encontrado foi a dissecção retrógrada como causa de óbito em cinco destes casos, mostrando que a técnica não foi suficiente para evitar a evolução natural nestes casos. Desta maneira, poassamos a empregar a técnica como cirurgia de complementação, ou $2:$ tempo, naqueles casos em que, após o tratamento cirúrgico convencional na dissecçāo Tipo A, permanece a dissecção distal. Não temos experiência pessoal com a presente técnica, mas sabemos por literatura que o Dr. Borst 
PALMA, H.; JULIANO, J. A.; CAL, R. G. R.; ALMEIDA, D. R.; OTA, L. H.; GIANOTTI, I. A.; ANDRADE, J. C. S.; BUFFOLO, E. - Tratamento dos aneurismas da aorta descendente por endoprótese (tromba de elefante). Rev. Bras. Cir. CardiovasC., 4(3): 190-194, 1989.

a tem usado também com 2: estágio de cirurgias, tanto nos aneurismas extensos de aorta, como na dissecção. Com a intenção de enriquecer mais a apresentação e não como crítico, gostariamos de fazer ao Dr. Palma três perguntas: 1) como vê o papel da dissecção retrógrada com a presente técnica, ou ela está abolida com a sutura proximal? 2) sabendo que os problemas relacionados à técnica são os embólicos, como foram contornados, na sua experiência? 3) considera a presente técnica melhor para tratamento de aneurismas verdadeiros, ou é indiferente sua indicação, também para as dissecções agudas? Parabenizamos o Dr. Palma e o grupo da Escola Paulista, pela importância deste trabalho e pela excelência de sua apresentação.

\section{DR. PAULO PRATES \\ Porto Alegre, RS}

Meus cumprimentos aos autores, pela importante contribuição ao tratamento das doenças da aorta. Meus agradecimentos à Comissão Organizadora do Congresso, pela oportunidade de poder comentá-lo. Os resultados do tratamento cirúrgico da dissecção aguda da aorta tipo B ou tipo III, conforme classificação de Stanford, ou de De Bakey, são caracterizados, tanto no passado como no presente, por alta mortalidade e morbidade. Diversas altenativas cirúrgicas têm sido sugeridas e mesmo antigas técnias, como a fenestração da aorta abdominal, têm sido citadas visando a um melhor resultado. A abordagem direta da aorta dissecada continua sendo um desafio à nossa especialidade e novas técnicas devem ser bem aceitas e ter seus resultados bem analisados. Tem sido nossa conduta, embora não uniforme, no Instituto de Cardiologia do Rio Grande do Sul, manter em tratamento clínico aqueles pacientes que respondam bem ao tratamento inicial. Indicamos cirurgia somente naqueles pacientes que não respondam ao tratamento nas primeiras horas, ou naqueles que apresentem as complicaçōes conhecidas. Evidentemente, isto nos leva a ter uma mortalidade cirúrgica mais elevada do que as apresentadas pelos grupos que indicam tratamento cirúrgico em todos os pacientes. No entretanto, se computarmos os resultados, tanto clínicos como cirúrgicos, nossa mortalidade fica semelhante, ou até menor que os dados apresentados na literatura. Por isso, continuamos com esta conduta. A técnica apresentada no trabaIho nos parece válida para os casos complicados, embora, a nosso ver, um acompanhamento mais longo seja necessário para sabermos se estes pacientes nāo irāo apresentar as complicaçōes que apresentam os pacientes não operados, numa incidência que varia em torno de $25 \%$. Gostariamos de perguntar aos autores em que fase os pacientes foram operados e se se tratava de casos com complicaçōes, ou de indicaçāo "eletiva".
Quanto aos aneurismas verdadeiros, nossa dúvida é se a introdução do tubo e o manuseio para isto necessário nāo poderiam causar embolias distais. Mais uma vez, maus cumprimentos aos autores. Obrigado.

\section{DR. ALEXANDER DOBRIANSKYJ Goiânia, GO}

Há alguns anos, estamos envolvidos na melhor opção sobre o tratamento cirúrgico de dissecções aórticas totais ou parciais. $O$ que nos chama atenção é o fato de se colocar apenas uma sutura na aorta e o tubo solto dentro da aorta. Achamos que o cuidado cirúrgico que se deve ter é refazer a luz verdadeira com tubo protético e readoçar sobre o mesmo as paredes da aorta sob visão direta. Nesse particular de aneurismas após a artéria subclávia esquerda (tipo B), em 12 pacientes operados com substituiçāo de aorta, sem óbitos, apenas dois (um aneurisma dissecante e outro verdadeiro) foram tratados de maneira, ao nosso ver, simples. Por toracotomia esquerda, clampeamento transversal da aorta antes da artéria subclávia e outro clampeamento abaixo, abertura longitudinal da aorta e colocação de uma endoprótese arterial de Dacron dotada de dois anéis sulcados metálicos para, por fora da aorta, serem amarrados com um "tape" umbilical, consegue-se rapidez, facilidade e, realmente, o readoçamento das paredes aórticas comprometidas. Achamos este um método mais simples. Obrigado.

\section{DR. PALMA (Encerrando)}

Gostaríamos, para finalizar, de agradecer os comentários dos Drs. Castilho, Prates e Dobrianskyj. A técnica apresentada, a nosso ver, constitui um avanço no tratamento dos aneurismas do tipo B e determinados saculares de aorta descendente. Os pacientes apresentados nesta casuística foram submetidos a cirurgia de urgência, não eletiva, pois apresentavam algum tipo de complicação. Gostariamos de realçar que, a nosso ver, esta cirurgia não é paliativa, pois a introduçāo da prótese na luz da aorta descendente tem como objetivo a excusão da lesão da intíma nos aneurismas tipo B e nos saculares do colo dos mesmos. Ainda com esta pequena casuística, não nos permitimos afirmar, com segurança, a evolução a longo prazo, assim como se algumas complicaçōes ocorridas foram, ou não, devidas à pequena experiência com a técnica. Porém, acreditamos ser muito boa a idéia de não abordarmos diretamente a aorta doente, ou manipularmos estruturas adjacentes como o pulmão. 